\title{
Vaporized Cannabis Is Effective and Well-Tolerated in an Adolescent with Tourette Syndrome
}

\author{
Natalia Szejko ${ }^{\mathrm{a}-\mathrm{c}}$ Ewgeni Jakubovski ${ }^{\mathrm{a}} \quad$ Carolin Fremer $^{\mathrm{a}} \quad$ Kirsten R. Müller-Vahla $^{\mathrm{a}}$ \\ ${ }^{a}$ Clinic of Psychiatry, Social Psychiatry and Psychotherapy, Hannover Medical School, Hannover, Germany; \\ ${ }^{b}$ Department of Neurology, Medical University of Warsaw, Warsaw, Poland; ' ${ }^{\circ}$ Department of Bioethics, Medical \\ University of Warsaw, Warsaw, Poland
}

\section{Keywords}

Tourette syndrome $\cdot$ Children · Medicinal cannabis .

Vaporization · Tetrahydrocannabinol

\begin{abstract}
We present the case of a 12-year-old boy diagnosed with Tourette syndrome, who was successfully treated with a combination of vaporized medicinal cannabis and oral pure tetrahydrocannabinol (THC). Due to severe motor tics resulting in insomnia, the parents - both of whom were medical doctors - decided to initiate treatment with $0.02 \mathrm{~g}$ vaporized cannabis (Bedrocan with a THC content of $22 \%$ and a cannabidiol content of $1 \%$; corresponding to a dose equivalent to $4.4 \mathrm{mg}$ THC) without prior consultation of a Tourette expert. This treatment resulted - according to the parents' report in an immediate and nearly complete remission of the tics. Due to a further increase in tics, the parents therefore decided to implement a regular treatment with a combination of vaporized medicinal cannabis (up to $0.1 \mathrm{~g}$ cannabis per day, varieties Bedrocan and Amnesia Haze, corresponding to $22 \mathrm{mg}$ THC/day) plus orally administered oil-based THC drops (maximum daily dose $=12.5 \mathrm{mg}$ THC) resulting in a marked tic reduction. During a visit in our clinic, we were able to observe the reported beneficial effects $30 \mathrm{~min}$ after
\end{abstract}

vaporization of $0.15 \mathrm{~g}$ cannabis (Amnesia Haze, equivalent to $33 \mathrm{mg}$ THC; in addition, $7 \mathrm{mg}$ oral THC were taken at home $6 \mathrm{~h}$ before the visit): tics, premonitory urges, and overall impairment significantly improved according to self-ratings, parent and clinician questionnaires. Importantly, no adverse events were reported. From this single case study, it is suggested that cannabis-based medicines and their combination (such as oral THC plus vaporized medicinal cannabis) are effective and safe in the treatment of severe tics in minors with TS. However, long-term follow-up is needed to confirm the beneficial treatment effects. We want to emphasize that in this boy treatment with cannabis was initiated by the parents before a consultation in our clinic has taken place. In our opinion, treatment with cannabis-based medicine in children should be regarded as a last-line treatment, when wellestablished treatments have failed to improve tics.

(c) 2019 The Author(s)
Published by S. Karger AG, Basel

\section{Introduction}

Tourette syndrome (TS) is a childhood-onset neurodevelopmental disorder characterized by motor and vocal tics. For the treatment of tics, behavioral therapy and antipsychotic medication are first-line treatments. In pa-

\begin{tabular}{ll}
\hline KARGER & $\begin{array}{l}\text { ( } 2019 \text { The Author(s) } \\
\text { Published by S. Karger AG, Basel }\end{array}$ \\
E-Mail karger@karger.com & $\begin{array}{l}\text { This article is licensed under the Creative Commons Attribution- } \\
\text { NonCommercial-NoDerivatives 4.0 International License (CC BY- } \\
\text { NC-ND) (http://www.karger.com/Services/OpenAccessLicense). } \\
\text { Usage and distribution for commercial purposes as well as any dis- } \\
\text { tribution of modified material requires written permission. }\end{array}$
\end{tabular}

Natalia Szejko

Clinic of Psychiatry, Social Psychiatry and Psychotherapy

Hannover Medical School, Carl-Neuberg-Strasse 1

DE-30625 Hannover (Germany)

E-Mail Szejko.Natalia@mh-hannover.de 
tients with severe and complex TS, who do not respond to these treatments, only few further treatment options can be offered including deep brain stimulation. Therefore, a substantial number of patients seek for alternative treatments including cannabis-based medicines (CBM). While there is increasing evidence that different CBM might be effective in the treatment of adults with TS [14], in minors only two single case reports are available suggesting beneficial effects of orally administered pure delta-9-tetrahydrocannabinol (THC) - the most psychoactive ingredient of cannabis - in 8- and 15-year-old boys with TS and comorbid attention-deficit/hyperactivity disorder $[5,6]$.

\section{Case Report}

We present the case of a 12-year-old boy diagnosed with TS, who was treated with a combination of medicinal cannabis and THC. We want to emphasize that treatment with cannabis was initiated by the parents before consultation in our clinic. Both the patient and parents consented to the publication of this case report. The boy's motor tics started at the age of 6 and vocal tics at the age of 10 years. Since then, his tics followed a typical waxing and waning course. The patient was diagnosed with TS for the first time at the age of 10 by his treating psychiatrist.

The family came self-motivated without referral to our TS outpatient clinic. At that time point, the patient was 12 years old and exhibited typical motor tics such as eye blinking, arm jerks, sticking out his tongue, abrupt leg and abdominal movements, and copropraxia as well as vocal tics such as squelching, throat clearing, rumbling, humming, and echolalia. Further psychiatric evaluation showed mild obsessive-compulsive behavior (touching objects and persons), sleeping problems, and mild self-injurious behavior with self-beating, but no evidence of comorbid attention-deficit/ hyperactivity disorder. He had never received any specific treatment for the tics besides relaxation techniques. At first presentation, the parents - both of whom were medical doctors, who had much experience in the treatment of their own patients with different CBM - reported that they had decided to medicate their son themselves with vaporized medicinal cannabis, rather than getting a prescription for an antipsychotic medication or any other kind of treatment or consulting a Tourette expert before. The decision was taken based on their son's suffering from severe tics and growing inability to fall asleep. So far, the boy had been treated twice with a single dose of $0.02 \mathrm{~g}$ cannabis (variety Bedrocan with a THC content of $22 \%$ and a cannabidiol content of $1 \%$; the dose was equivalent to $4.4 \mathrm{mg}$ THC). According to the parents' report, both administrations resulted in an immediate and nearly complete remission of tics so that the boy was able to fall asleep without problems. Neither after the intake nor on the following day did any adverse event occur according to the patient's and his parents' report. In order to assess the described beneficial treatment effects of medicinal cannabis in a clinical environment, we invited the family to come for a second visit.

During the second visit at our clinic 1.5 months later, the parents reported about a marked deterioration of tics over the last few weeks with severe motor tics resulting in headache as well as intense and loud screaming so that - for the first time - the parents felt compelled not to send the boy to school. Therefore, the parents decided in favor of a regular treatment with vaporized medicinal cannabis (taken at home) with a maximum dose of $0.1 \mathrm{~g}$ cannabis per day (Bedrocan and Amnesia Haze varieties both containing $22 \%$ of THC) corresponding to $22 \mathrm{mg}$ of THC. In addition, they decided to introduce treatment with oral THC (oil-based drops) with a maximum daily dose of $12.5 \mathrm{mg}$ THC divided into two doses (taken outside the house). Thus, maximum daily dosage of THC was $34.5 \mathrm{mg}$ according to the parents' report.

\section{Results}

During that second visit, the patient presented an identical tic repertoire as described during the first consultation; however, on that day, he had already received medication ( $7 \mathrm{mg}$ THC) $6 \mathrm{~h}$ before the appointment. Both the parents and the patient reported not only about a tic reduction, but also improved concentration at school after having started regular treatment with CBM. In addition, both reported that they could clearly differentiate between clinical effects related to intake of oral THC and vaporized medicinal cannabis, respectively: while oral THC had a superior effect on tics and sleep compared to cannabis, vaporized medicinal cannabis resulted in an immediate remission of headache due to the tics. The effect of oral THC lasted longer compared to the effect of vaporized cannabis. Absolutely no adverse events were reported neither by the patient nor his parents.

In order to assess immediate effects of vaporized cannabis in this boy, we performed a variety of clinical assessments before and about $30 \mathrm{~min}$ after vaporization of $0.15 \mathrm{~g}$ cannabis (Amnesia Haze, equivalent to $33 \mathrm{mg}$ THC; at that time, the boy reported a maximum effect; for details, see Table 1). In summary, inhalation of cannabis resulted in an immediate and marked reduction of both tics, premonitory urges, and overall impairment across different observers (self, parents, and clinician). No adverse events were reported or observed.

\section{Discussion/Conclusion}

While there is increasing evidence that CBM is effective in the treatment of a variety of conditions in adult populations [7], only very little is known about efficacy and safety of CBM in children and adolescents [8]. In a recent systematic review, only 22 studies have been identified using CBM in minors, most commonly pure THC 
Table 1. Clinical assessments before (baseline) and after an interval of $30 \mathrm{~min}$ following cannabis inhalation (follow-up) in combination with oral THC

\begin{tabular}{llrl}
\hline Symptom & Scale (range) & Baseline & Follow-up \\
\hline Tics & Total tic score of the Yale Global Tic Severity Scale (0-50) & 29 & 19 \\
& Rush Video-Based Tic Rating Scale (0-20) & 17 & 56 \\
& Parent Tic Questionnaire (0-224) & 30 & 13 \\
Impairment & Impairment score of the Yale Global Tic Severity Scale (0-50) & 59 & 29 \\
Tics + impairment & Yale Global Tic Severity Scale, global score (0-100) & 23 & 15 \\
Premonitory urge & Premonitory Urge for Tics Scale (0-40) & 42 \\
Quality of life & Gilles de la Tourette Syndrome - Quality of Life Scale (0-100) & 2 \\
& Visual Analogue Scale of Gilles de la Tourette Syndrome - Quality of Life Scale & 45 & 85 \\
Global impairment & Clinical Global Impression - Severity Scale (0-7) & 3 \\
& Clinical Global Impression - Improvement Scale (0-7) & 2 \\
\hline
\end{tabular}

and cannabidiol [8]. To the best of our knowledge, this is not only the first case study reporting about beneficial effects of vaporized cannabis with high THC content in a boy with TS, but the first such report ever in a child.

In open uncontrolled case studies including more than 200 patients, adult patients with TS often report about beneficial effects of inhaled cannabis [9]. In this case study, both the family's report as well as the expert clinical ratings suggested that not only THC - as described earlier [8] - but also vaporized cannabis are effective in the reduction of tics and premonitory urges. Remarkably, even relatively high doses of THC were well tolerated and even improved concentration according to the patient's report. This observation of good tolerance of CBM is completely in line with reports of good tolerance in studies of CBM treatment as a prevention of vomiting due to antineoplastic treatment $[10,11]$ and CBM treatment of treatment-resistant spasticity [12] in pediatric populations. However, based on the short-term treatment in this single case, no statement can be made about long-term (side) effects of cannabis and THC in children and adolescents with TS.

Since tics typically wax and wane over time, demonstrate an age dependency with a maximum at age 10-12 years, and are influenced by environmental factors, it cannot be excluded that in this boy improvement of tics and other symptoms was related to these factors instead of treatment with CBM.

Although there is increasing evidence that CBM might be effective in the treatment of several different conditions, medical doctors often hesitate to use CBM due to concerns of long-term side effects including increased risk of psychosis and cognitive impairment. However, in a recent meta-analysis [13], it could be demonstrated that in most studies in youth the magnitude and persistence of cognitive deficits of cannabis have been overstated. In the authors' opinion, reported deficits reflect residual effects from acute use or withdrawal rather than persistent cognitive deficits. In contrast, it is generally accepted that children who start using cannabis before the age of 15 are at increased risk of psychotic disorders [14]. However, until today it is unclear whether observations in healthy children using high doses of skunk-like cannabis recreationally can be transferred to children suffering from neuropsychiatric disorders including TS, who use medicinal cannabis supervised by a medical doctor. Although the database is very limited, so far, increased risk of psychosis has not been reported in pediatric patients treated with CBM [10-12].

It should be noted that in this boy treatment with vaporized medicinal cannabis was not initiated and prescribed by the authors, but by the parents before the first presentation in our clinic. This was possible, because both were medical doctors. Keeping in mind that to date only very little is known about the effects of CBM in children, we do not recommend the use of vaporized cannabis in children with TS. However, in the authors' opinion, in severely affected and otherwise treatment-resistant children, treatment with other orally taken CBM such as pure THC or cannabis extracts should be taken into consideration as a rescue therapy, before thinking about surgical treatment with deep brain stimulation.

\section{Statement of Ethics}

The authors have no ethical conflicts to disclose. The patient's parents gave written informed consent in accordance with the Declaration of Helsinki. 


\section{Disclosure Statement}

K.R.M.-V. has nonfinancial competing interests as a member of the TAA medical advisory board, the scientific advisory board of the German Tourette Association TGD, the board of directors of the German (ACM) and the International (IACM) Association for Cannabinoid Medicines, and the committee of experts for narcotic drugs at the federal opium bureau of the Federal Institute for Drugs and Medical Devices (BfArM) in Germany; has received financial or material research support from the EU (FP7HEALTH-2011 No. 278367, FP7-PEOPLE-2012-ITN No. 316978), the German Research Foundation (DFG: GZ MU 1527/3-1), the German Ministry of Education and Research (BMBF: 01KG1421), the National Institute of Mental Health (NIMH), the Tourette Gesellschaft Deutschland e.V., the Else-Kroner-Fresenius-Stiftung, and GW, Almirall, Abide Therapeutics, and Therapix Biosiences; has served as a guest editor for Frontiers in Neurology on the research topic "The neurobiology and genetics of Gilles de la Tourette syndrome: new avenues through large-scale collaborative projects," is an associate editor for "Cannabis and Cannabinoid Research" and an Editorial Board Member of "Medical Cannabis and Cannabinoids"; has received consultant's honoraria from Abide Therapeutics, Fundacion Canna, and Therapix Biosiences, speaker's fees from Tilray, and royalties from Medizinisch Wissenschaftliche Verlagsgesellschaft Berlin, and is a consultant for Zynerba Pharmaceuticals.

\section{Funding Sources}

The authors report no funding sources.

\section{Author Contributions}

Research project: (a) conception (K.R.M.-V.), (b) organization (K.R.M.-V.), (c) execution (K.R.M.-V., N.S., C.F.). Clinical examination (K.R.M.-V., N.S., C.F.). Manuscript preparation: (a) writing of the first draft (K.R.M.-V., N.S.), (b) review and critique (K.R.M.-V., E.J., N.S., C.F.).

\section{References}

1 Abi-Jaoude E, Chen L, Cheung P, et al. Preliminary Evidence on Cannabis Effectiveness and Tolerability for Adults With Tourette Syndrome. J Neuropsychiatry Clin Neurosci. 2017 Fall;29(4):391-400.

2 Müller-Vahl KR, Schneider U, Prevedel H, et al. Delta 9-tetrahydrocannabinol (THC) is effective in the treatment of tics in Tourette syndrome: a 6-week randomized trial. J Clin Psychiatry. 2003 Apr;64(4):459-65.

3 Trainor D, Evans L, Bird R. Severe motor and vocal tics controlled with Sativex ${ }^{\circledR}$. Australas Psychiatry. 2016 Dec;24(6):541-4.

4 Kanaan AS, Jakubovski E, Müller-Vahl K. Significant tic reduction in an otherwise treatment-resistant patient with gilles de la tourette syndrome following treatment with nabiximols. Brain Sci. 2017 Apr;7(5):E47.

5 Hasan A, Rothenberger A, Münchau A, Wobrock T, Falkai P, Roessner V. Oral delta 9-tetrahydrocannabinol improved refractory Gilles de la Tourette syndrome in an adolescent by increasing intracortical inhibition: a case report. J Clin Psychopharmacol. 2010 Apr;30(2):190-2.

6 Szejko N, Jakubovski E, Fremer C, Kunert K, Mueller-Vahl K. Delta-9-tetrahydrocannabinol for the treatment of a child with Tourette syndrome: case report. EJMCR. 2018 May;2: 39-41.

7 Abrams DI. The therapeutic effects of Cannabis and cannabinoids: An update from the National Academies of Sciences, Engineering and Medicine report. Eur J Intern Med. 2018 Mar;49:7-11.

8 Wong SS, Wilens TE. Medical Cannabinoids in Children and Adolescents: A Systematic Review. Pediatrics. 2017 Nov; 140(5): e20171818.

9 Müller-Vahl KR, Kolbe H, Schneider U, Emrich HM. Cannabinoids: possible role in patho-physiology and therapy of Gilles de la Tourette syndrome. Acta Psychiatr Scand. 1998 Dec;98(6):502-6.

10 Abrahamov A, Abrahamov A, Mechoulam R. An efficient new cannabinoid antiemetic in pediatric oncology. Life Sci. 1995;56(23-24): 2097-102.

11 Elder JJ, Knoderer HM. Characterization of Dronabinol Usage in a Pediatric Oncology Population. J Pediatr Pharmacol Ther. 2015 Nov-Dec;20(6):462-7.

12 Kuhlen M, Hoell JI, Gagnon G, Balzer S, Oommen PT, Borkhardt A, et al. Effective treatment of spasticity using dronabinol in pediatric palliative care. Eur J Paediatr Neurol. 2016 Nov;20(6):898-903.

13 Scott JC, Slomiak ST, Jones JD, Rosen AF, Moore TM, Gur RC. Association of cannabis with cognitive functioning in adolescents and young adults: A systematic review and metaanalysis. JAMA Psychiatry. 2018 Jun;75(6): 585-95.

14 Di Forti M, Marconi A, Carra E, Fraietta S, Trotta A, Bonomo M, et al. Proportion of patients in south London with first-episode psychosis attributable to use of high potency cannabis: a case-control study. Lancet Psychiatry. 2015 Mar;2(3):233-8. 